\title{
Le briefing-débriefing : une procédure pour lever les barrières pesant sur l'apprentissage organisationnel ?
}

Cet article interroge la façon dont les organisations gèrent les facteurs défavorables à l'apprentissage organisationnel. S'inscrivant dans le cadre des théories de l'apprentissage dans et par l'action, la question posée dans cette contribution est la suivante : comment les procédures de briefing-débriefing permettent-elles de lever les barrières pesant sur l'apprentissage organisationnel ? L'analyse d'une étude de cas réalisée au sein de différents escadrons de l'Armée de l'air révèle que le briefing-débriefing repose sur une variété de mécanismes d'apprentissage, de valeurs, d'attitudes comportementales et d'artefacts technologiques, dont l'articulation est enactée par les acteurs afin de favoriser l'apprentissage. Ensemble, ces ressources permettent aux équipes de lever les barrières techniques, socioculturelles et psychologiques pesant sur l'apprentissage organisationnel.

\section{Introduction}

Les théories de l'apprentissage dans et par l'action (ARGYRIS et SCHÖN, 1978) sont régulièrement mobilisées pour analyser les phénomènes d'apprentissage organisationnel. Enracinées dans une épistémologie pragmatiste de la connaissance (JAMES, 1912 ; DEWEY, 1938), elles « conçoivent l'apprentissage organisationnel comme un processus itératif d'action et de réflexion " (EDMONDSON, 2002, p. 128). L'apprentissage organisationnel repose ainsi sur trois piliers : une connaissance fondamentalement expérientielle, un processus social d'enquête et de réflexion critiques ayant pour objet l'action et ses conséquences et, enfin, des expériences individuelles attachées aux situations de travail, dans lesquelles les actions entreprises par les individus sont à la fois le sujet et le produit de l'apprentissage (ORMEROD, 2006).

Plusieurs facteurs peuvent influencer l'apprentissage organisationnel dans et par l'action. Cannon et Edmondson (2005) insistent notamment sur la complexité des systèmes techniques, sur la nature des compétences des individus et sur le degré de tolérance de l'organisation vis-à-vis de l'erreur. D'autres auteurs mettent en avant l'influence des facteurs psychologiques et organisationnels. Carmeli (2007) suggère, par exemple, que l'absence d'un sentiment de sécurité face à l'erreur constitue une condition défavorable à l'accumulation de l'expérience au niveau organisationnel. L'entretien d'un climat de sécurité psychologique est indispensable à l'établissement de relations interpersonnelles saines sans lesquelles les émotions des acteurs (stress, peur, anxiété) peuvent devenir des barrières à l'apprentissage (CATINO et PATRIOTTA, 2013).

Si elles permettent d'identifier les facteurs favorables ou défavorables à l'apprentissage organisationnel, ces contributions n'abordent qu'indirectement la question de leur gestion par les équipes qui composent l'organisation. Or, ces équipes offrent des espaces d'interaction propices au partage des connaissances expérientielles, elles sont le " lieu » où les individus s'engagent dans l'analyse réflexive des déterminants de leurs actions et des conséquences de celles-ci. II est donc essentiel de comprendre la manière dont les procédures d'apprentissage mises en œuvre par les équipes leur permettent de gérer les multiples facteurs favorables ou, au contraire, défavorables à l'apprentissage. 
Dans ce cadre, les procédures de briefing-débriefing utilisées dans les milieux professionnels de l'aéronautique civile, de la Défense, de la sécurité civile, de la police ou de la médecine hospitalière sont intéressantes pour illustrer notre propos. Ces procédures dites de retour d'expérience (BES 1998 ; VAN DER VEGT et BUNDERSON, 2005 ; GODÉ et BARBAROUX, 2012 ; FROMIGUÉ et al., 2015) encouragent en effet l'examen systématique et répété des erreurs commises par l'équipe et/ou par ses membres. Elles favorisent la construction du sens collectif (GODÉ, 2012) et produisent une amélioration significative de la performance opérationnelle. Elles offrent donc un matériau empirique à partir duquel est étudiée la gestion des facteurs qui ont une influence sur l'apprentissage organisationnel.

La question posée dans cette contribution est la suivante : comment les procédures de briefingdébriefing permettent-elles de lever les barrières pesant sur l'apprentissage organisationnel ? Pour répondre à cette question, nous développerons une étude de cas portant sur la procédure du briefing-débriefing mis en $œ u v r e$ par les équipages navigants (pilotes, navigateurs et/ou mécaniciens) de l'Armée de l'air française.

Nous présenterons ensuite les facteurs qui ont une influence sur l'apprentissage dans et par l'action, en particulier l'apprentissage par l'erreur, après quoi nous décrirons la méthodologie de l'étude de cas que nous avons réalisée au sein du milieu aéronautique militaire français. Enfin, les résultats de cette étude et leurs implications théoriques et managériales seront présentés.

\section{Les processus d'apprentissage dans et par l'action : définitions et facteurs défavorables}

Le sujet de l'apprentissage organisationnel dans et par l'action a inspiré de nombreux travaux. Désignant le processus de réflexion sur un travail réalisé par soi-même et par d'autres, l'apprentissage au sens pragmatiste du terme est conduit sous le regard critique de ses pairs. II permet de tirer de nouveaux enseignements concernant les actions entreprises et les raisons qui les ont motivées, et de résoudre en temps réel les problèmes qui se présentent à l'organisation (REVANS, 1982 ; KOLB, 1984). Dans ce cadre, l'expérience doit être éprouvée pour pouvoir être à la fois l'objet et le produit de l'apprentissage.

Selon Cannon et Edmondson (2005), l'apprentissage organisationnel repose sur trois activités : l'identification d'une déviation entre l'action prescrite ou planifiée et le résultat de l'action, l'analyse des causes de cette déviation et l'expérimentation délibérée de situations favorables à l'apparition de ces déviations. Celles-ci sont assimilables à des erreurs. Elles sont définies comme " des écarts non intentionnels relatifs à des règles ou à des procédures (...) qui peuvent potentiellement avoir des conséquences organisationnelles contrariantes " (GOODMAN et al., 2011, p. 152). Dans ce cadre, l'attitude des individus, l'usage des technologies facilitant la collecte, le stockage et la diffusion des informations d'activité et l'ensemble des dispositifs mis en œuvre par l'organisation pour analyser les causes de ces déviations sont importants : en effet, ils conditionnent la capacité d'apprentissage des équipes. Putz et al. (2013) suggèrent que les définitions de l'erreur se partagent en deux groupes : le premier concerne l'erreur individuelle définie comme un résultat non anticipé d'actions conduisant à un échec ; le second fait référence à une déviation par rapport à une norme, une routine, un standard ou un but fixé par l'organisation. Dans la pratique, l'erreur individuelle et l'erreur par rapport à la norme peuvent se confondre. En 1996, Vaughan parle même de normalisation de la déviance pour expliquer comment au sein de la NASA des écarts par rapport à la performance attendue aient pu être interprétés comme conformes aux procédures de sécurité, conduisant ainsi à autoriser le lancement de la navette Challenger en dépit du mauvais fonctionnement d'un joint sur l'un de ses propulseurs (LAROCHE, 1998).

Il apparaît ainsi que l'apprentissage organisationnel dans et par l'action relève de la capacité des individus et des équipes d'apprendre de leurs erreurs. II s'agit d'" une activité qui demande des efforts et qui permet aux individus de réfléchir aux erreurs qu'ils ont commises, d'en comprendre les causes et de développer de nouvelles connaissances susceptibles d'être appliquées pour des actions et des décisions futures » (CATINO et PATRIOTTA, 2013, p. 437). Dans cette perspective, les organisations performantes en matière d'apprentissage sont celles qui sont capables de favoriser l'expérimentation délibérée de l'erreur en créant les conditions propices à l'expression du sens critique des acteurs (CANNON et EDMONDSON, 2005).

En pratique, la mise en œuvre des procédures d'apprentissage organisationnel ne va pas de soi. Certains facteurs peuvent entraver l'implication des individus et des équipes dans un processus d'enquête réflexive et d'analyse critique de leurs actions (CARMELI, 2007 ; ZHAO, 2011). De nombreux facteurs techniques, organisationnels, culturels et psychologiques sont susceptibles de limiter l'apprentissage (CANNON et EDMONDSON, 2005 ; ZHAO, 2011). Ainsi, par exemple, Catino et Patriotta (2013) démontrent les bénéfices des approches pragmatistes de l'apprentissage à l'échelle de l'organisation (améliorer la sûreté, la fiabilité et la résilience organisationnelles) à condition de tenir compte de certains biais ou de certains freins émotionnels, organisationnels et culturels. Dans ce cadre, la culture de l'organisation vis-à-vis de l'erreur oriente la façon dont les équipes agissent et réagissent sur le double plan cognitif et émotionnel. De leur côté, Ron et al. (2006) insistent sur les fonctions sociales et psychologiques nécessaires à l'apprentissage dans et par l'action. Les premières incluent la discipline, les relations émotionnelles et cognitives des individus vis-à-vis de l'erreur, le contrôle de la performance par la hiérarchie ou encore la communication des intentions du commandement. Les secondes, quant à elles, renvoient à la capacité de résilience des individus 
face à leurs erreurs, au système de reconnaissance et de récompense à l'œuvre dans l'organisation et à la régulation sociale. De fait, les valeurs organisationnelles peuvent ne pas être alignées sur les exigences d'un apprentissage dans et par l'action décourageant ainsi la réflexion collective et perturbant l'attribution des responsabilités et la reconnaissance des erreurs. Ces valeurs organisationnelles peuvent également limiter le partage d'un sentiment de sécurité, l'esprit de dialogue et la capacité d'écoute des acteurs.

Le Tableau 1 ci-dessous synthétise les facteurs le plus souvent cités dans la littérature comme étant susceptibles de contrarier le processus d'apprentissage pragmatiste.

Nous développerons ci-après une étude de cas décrivant les procédures de briefing-débriefing mises en œuvre par les équipages navigants au sein des escadrons de l'Armée de l'air française. Elle nous permettra d'illustrer comment ce dispositif d'origine militaire permet de lever les facteurs défavorables à l'apprentissage organisationnel.

\section{Présentation de l'étude de cas : les procédures de briefing-débriefing dans les escadrons de l'Armée de l'air française}

Ce travail de recherche repose sur une étude de cas exploratoire (YIN, 2003) portant sur les pratiques d'acteurs impliqués dans un processus d'apprentissage de type briefing-débriefing. Plus précisément, l'attention se porte sur les débriefings auxquels participent quotidiennement (après chaque vol) les équipages des escadrons de chasse, de transport, de formation et de présentation (Patrouille de France - PAF -, et équipe de voltige de l'Armée de l'air - EVAA) de l'Armée de l'air française.

Le contexte de notre recherche : équipages, missions et erreurs dans le milieu aéronautique militaire

La journée de travail d'un escadron commence toujours par un briefing général d'une durée d'envi- ron 30 minutes réunissant l'ensemble des personnels navigants ayant un ou plusieurs vols planifiés. À l'issue de ce briefing général, ils se séparent pour former des équipages se composant de deux à quatre membres afin de participer à un briefing dédié $\mathrm{au}(\mathrm{x})$ vol(s) qu'ils devront effectuer. Immédiatement après ce vol (ou ces vols), ils se retrouvent pour un débriefing de mission qui dure de 40 à 45 minutes. Cette procédure générale de " briefing-débriefing » concerne l'ensemble des personnels navigants de l'Armée de l'air française, et ce, alors même que leurs compétences et leurs missions sont sensiblement différentes selon les escadrons (voir la Figure 1 de la page suivante).

Très formelles, les sessions de débriefing suivent des procédures standard formalisées dans un manuel de I'OTAN. II s'agit de prendre à rebours l'ensemble des points évoqués durant le briefing, en insistant sur la sécurité du vol. La plupart des escadrons de chasse bénéficient de systèmes de reconstitution des vols reposant sur la numérisation des trajectoires des aéronefs. En ce qui concerne les escadrons de présentation (PAF et EVAA), ceux-ci sont toujours accompagnés d'un caméraman qui filme les vols et transmet ensuite le document aux pilotes sous une forme numérisée. Ces escadrons ont ainsi accès à une représentation claire de leur formation et des exercices réalisés durant le vol, et sont donc en mesure d'évaluer sans ambiguïté la qualité de la mission qu'ils ont effectuée. Chaque vol est scruté avec attention, une insistance particulière étant apportée aux erreurs, à leur(s) cause(s) et aux solutions qu'il aurait été opportun de mettre en œuvre. Une tension certaine caractérise ces moments de débriefing, qui tient au fait que les critiques, la plupart du temps sévères et cassantes, sont faites en public. Finalement, les pilotes en formation subissent une pression supplémentaire durant les séances de débriefing, dans la mesure où ils sont évalués par leur moniteur instructeur, qui émet ensuite un avis formel sur leur progression.

Dans le cadre d'un vol, une « erreur » peut avoir des conséquences plus ou moins graves. II peut s'agir, par exemple, d'un manquement aux règles de la sécurité des vols (qui sont toujours rappelées durant le briefing), comme un vol à basse altitude au-dessus d'habitations, mais elle peut également faire référence à une erreur

\begin{tabular}{|l|l|l|}
\hline \multicolumn{1}{|c|}{ Facteurs défavorables } & \multicolumn{1}{|c|}{ Illustrations } & \multicolumn{1}{c|}{ Auteurs } \\
\hline Techniques & $\begin{array}{l}\text { Complexité des systèmes, } \\
\text { compétences absentes ou } \\
\text { insuffisantes }\end{array}$ & $\begin{array}{l}\text { Edmondson et Cannon (2005); } \\
\text { Lipshitz et al. (2002) }\end{array}$ \\
\hline $\begin{array}{l}\text { Socioculturels (normes sociales et } \\
\text { valeurs individuelles) }\end{array}$ & $\begin{array}{l}\text { Pénalisation de l'erreur, absence } \\
\text { de culture du dialogue, peu de } \\
\text { réflexivité, dévalorisation de } \\
\text { l'enquête }\end{array}$ & $\begin{array}{l}\text { Edmondson (2002); Edmondson } \\
\text { et Cannon (2005); Revans } \\
(1982) ; \text { Kolb (1983) }\end{array}$ \\
\hline Psychologiques et émotionnels & $\begin{array}{l}\text { Stress, peur, anxiété, culpabilité, } \\
\text { insécurité, mauvaise estime de soi }\end{array}$ & $\begin{array}{l}\text { Zhao (2011); Catino et Patriotta } \\
(2013) \text {; Ron et al. (2006); Carmeli } \\
(2007)\end{array}$ \\
\hline
\end{tabular}

Tableau 1 : Les principaux facteurs défavorables au processus d'apprentissage pragmatiste. 


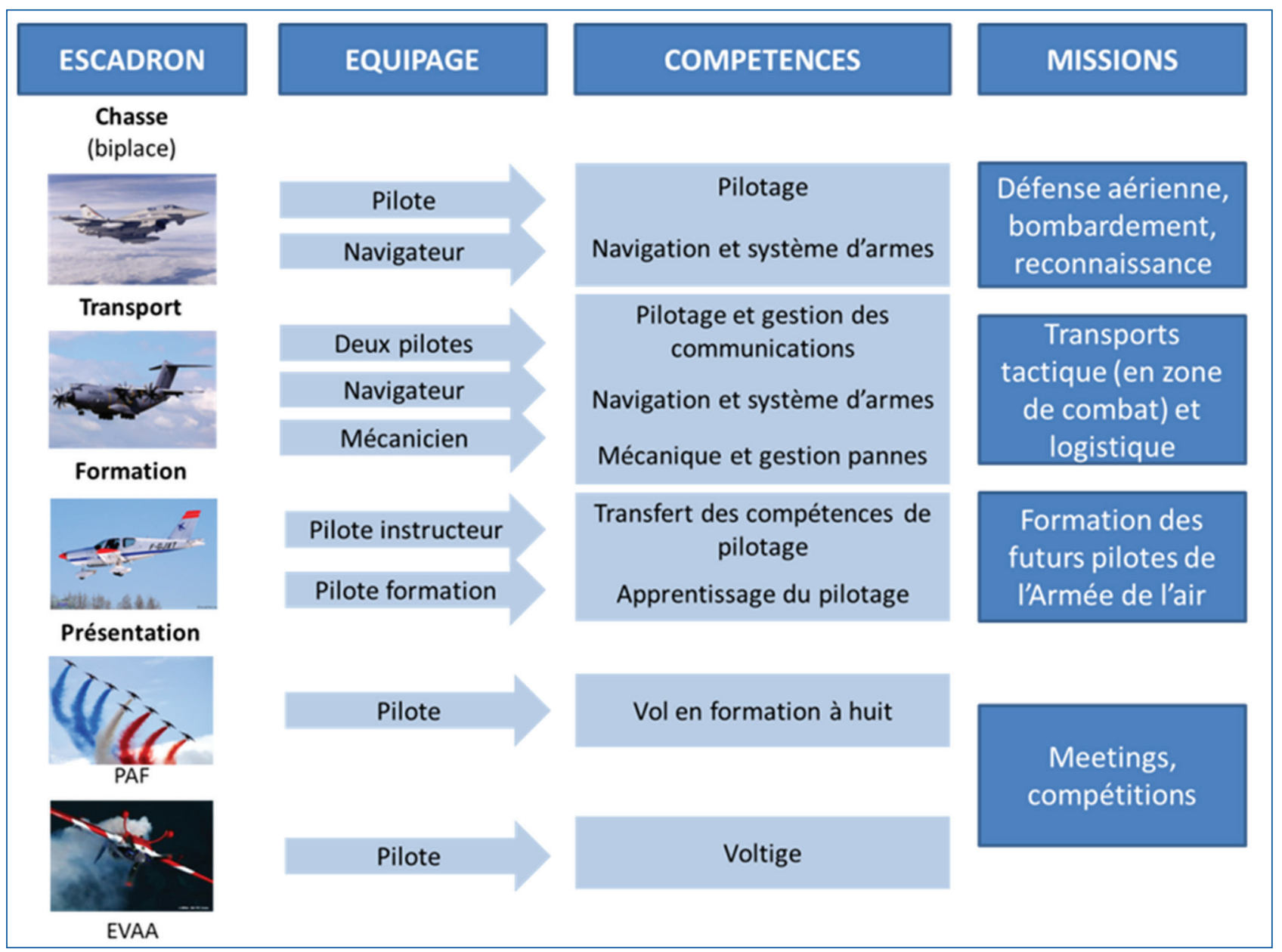

Figure 1 : Les différents escadrons de l'Armée de l'air et leurs missions.

de pilotage pouvant mettre en péril son propre appareil ou celui de son co-équipier et conduire à un incident ou à un accident. Le Tableau 2 de la page suivante propose quelques illustrations d'erreurs qui ont été commises au sein des quatre escadrons sur lesquels a porté notre étude de cas. Les données sont issues de rapports publiés en ligne par les bureaux enquêtes accidents-Défense (BEAD).

\section{Méthodologie}

Le corpus des données de terrain a été recueilli sur une période de deux ans et demi. II a été construit par triangulation (EISENHARDT, 1989) : seize entretiens individuels semi directifs ont été conduits auprès de pilotes et de navigateurs affectés à deux escadrons de chasse (Dijon et Nancy) et quatre moniteurs de l'escadron de formation de Salon-de-Provence ont également été entendus. Huit entretiens individuels ont été réalisés au sein de deux escadrons de transport (Evreux et Orléans) auprès de pilotes, de navigateurs et de mécaniciens navigants, et un entretien collectif a été organisé. Nous avons aussi interviewé six pilotes de la PAF (sur les neuf pilotes que compte la patrouille), les six pilotes de l'EVAA et deux mécaniciens (sur les treize membres que compte l'équipe).

Les entretiens ont duré une heure, en moyenne. Ils ont été enregistrés, puis retranscrits. Des observations non participatives ont été réalisées au cours de deux séances de briefing général dans les escadrons de chasse et durant deux autres séances de briefing (appelé " musique ») dans les escadrons de présentation.

Nous avons également assisté à sept séances de débriefing dans les différents escadrons. Durant ces observations, des notes manuscrites ont été prises ainsi que des photos. Une attention particulière a été apportée à la façon dont les débriefings étaient organisés (espace, technologies, manuels, participants) et conduits (pratiques de communication verbales et non verbales, contenu et nature des discussions engagées). Enfin, une analyse des guides et des procédures de débriefing suivis par les escadrons visités, ainsi que l'examen d'une dizaine de documents non classifiés français, anglo-américains et OTAN relatifs à cet exercice, ont été effectués.

Le traitement des données de terrain a été réalisé par codage ouvert afin de faire émerger les thèmes représentatifs et récurrents au fil de l'analyse. Ce traitement portait sur les transcriptions des entretiens, les notes de terrain prises lors des observations in situ consignant le déroulement des tâches exécutées lors des briefings et des débriefings, ainsi que sur certains passages des documents internes. Opéré dans la perspective de comprendre la façon dont les séances de briefing-débriefing sont réalisées au sein des 


\begin{tabular}{|c|c|c|c|}
\hline Escadron & Date et source & Exemple d'erreurs & Cause(s) de l'erreur \\
\hline Chasse & $\begin{array}{l}\text { Mars } 2014 \\
\text { BEAD-air-A-2014- } \\
\text { 008-I }\end{array}$ & $\begin{array}{l}\text { Lors du retour sur un terrain } \\
\text { d'atterrissage, deux Mirage } 2000 \\
\text { se présentent sur le taxiway (la } \\
\text { voie de circulation entre la piste } \\
\text { et le tarmac) en même temps } \\
\text { et en sens opposés. Les deux } \\
\text { avions s'immobilisent à environ } \\
200 \text { mètres l'un de l'autre. }\end{array}$ & $\begin{array}{l}\text { Une conscience de la situation } \\
\text { erronée notamment due au } \\
\text { fait que la mission n'était pas } \\
\text { initialement prévue pour un des } \\
\text { pilotes et que sa préparation était } \\
\text { insuffisante. }\end{array}$ \\
\hline Transport & $\begin{array}{l}\text { Avril } 2010 \\
\text { BEAD-air-A-2010- } \\
006-I\end{array}$ & $\begin{array}{l}\text { Au cours d'un exercice durant } \\
\text { lequel un aéro-largage de } \\
\text { parachutistes doit être réalisé, un } \\
\text { ensemble de décisions en cockpit } \\
\text { conduit le pilote à effectuer un } \\
\text { virage serré par la droite. Les } \\
\text { parachutistes qui se préparaient } \\
\text { pour le saut imminent chutent. }\end{array}$ & $\begin{array}{l}\text { L'absence de définition préalable } \\
\text { de conventions d'exercice, } \\
\text { notamment lors du briefing } \\
\text { mission, n'a pas incité le pilote } \\
\text { à modérer l'exécution de la } \\
\text { manœuvre. }\end{array}$ \\
\hline Formation & $\begin{array}{l}\text { Mars } 2012 \\
\text { BEAD-air-A-2012- } \\
\text { 004-I }\end{array}$ & $\begin{array}{l}\text { Lors d'un exercice de poser- } \\
\text { décoller, l'avion de formation se } \\
\text { pose train non sorti sur le terrain } \\
\text { d'atterrissage. L'équipage a oublié } \\
\text { d'abaisser la commande du train } \\
\text { d'atterrissage }\end{array}$ & $\begin{array}{l}\text { Le pilote en instruction a } \\
\text { consacré toutes ses ressources } \\
\text { attentionnelles à l'approche et à la } \\
\text { vitesse. La vigilance du moniteur } \\
\text { n'était pas suffisante. }\end{array}$ \\
\hline $\begin{array}{l}\text { Présentation } \\
\text { (PAF) }\end{array}$ & $\begin{array}{l}\text { Avril } 2010 \\
\text { BEAD-air-A-2010- } \\
\text { 005-A }\end{array}$ & $\begin{array}{l}\text { Durant la réalisation d'une figure } \\
\text { en formation, un pilote perd } \\
\text { le contrôle de son appareil et } \\
\text { s'éjecte à proximité immédiate du } \\
\text { sol. }\end{array}$ & $\begin{array}{l}\text { Perte de contrôle résultant d'une } \\
\text { sortie du domaine de vol de } \\
\text { l'avion, notamment due: } \\
\text { - 1) à une figure ayant été modi- } \\
\text { fiée, réduisant les marges d'évo- } \\
\text { lution en sortie d'exécution, } \\
\text { - et 2) à une interruption de l'en- } \\
\text { traînement hivernal engendrant } \\
\text { un retard dans l'assimilation de la } \\
\text { figure par le pilote. }\end{array}$ \\
\hline
\end{tabular}

Tableau 2 : Exemples d'erreurs examinées par le BEAD.

escadrons de l'Armée de l'air française, le codage des données révèle trois catégories thématiques principales : a) les mécanismes d'apprentissage à l'œuvre, b) les fonctionnalités des technologies utilisées et c) les valeurs sociales et les comportements individuels.

\section{Résultats de terrain}

Les mécanismes d'apprentissage : apprendre par l'erreur, par l'expérimentation et par les autres À la question "quels sont selon vous les objectifs principaux poursuivis par le briefing-débriefing ? ", l'ensemble des personnels interviewés répond: « favoriser l'apprentissage et la progression individuelle, d'une part, et permettre au collectif de s'améliorer, d'autre part ».

La première fonction des séances de briefing-débriefing est de favoriser l'entraînement et l'apprentissage individuels. Les pilotes et les navigateurs sont méthodiquement évalués par leurs collègues et/ou leur hiérarchie (en ce qui concerne les pilotes) et ils peuvent être sanctionnés, en cas d'échec (mauvaise décision, non-respect des procédures de sécurité des vols, etc.). Comme le précise un pilote de chasse :

« Le premier intérêt du débriefing, c'est de pouvoir s'améliorer. C'est au travers du débriefing que l'on va pouvoir analyser ce qui a été fait efficacement ou non, et trouver des solutions pour ce que l'on n'a peut-être pas fait efficacement en vol - à froid, en analysant ce qui s'est passé, pour que la prochaine fois on ait les bonnes solutions, rapidement... »

Tous les personnels navigants s'accordent sur cette fonction élémentaire du débriefing et considèrent que la détection et la correction des erreurs sont les meilleures façons de progresser et de devenir performant. Si les pilotes en cours d'instruction doivent particulièrement s'impliquer, les « seniors " sont également concernés. Ils reconnaissent volontiers que même le plus expérimenté et talentueux des pilotes n'est pas à l'abri d'une erreur et qu'il retire forcément avantage des commentaires et des remarques formulés par un pair. Plusieurs 
de nos interviewés nous ont précisé que l'examen critique de ce qui a été réalisé durant le vol représente l'essence même du débriefing. Ainsi, l'un d'entre eux nous explique que :

"Le débriefing est essentiellement axé sur savoir ce qui s'est passé au cours de la mission - exactement. Pourquoi ? Parce qu'en l'air, ça va trop vite... C'està-dire, retracer un peu (presque comme un travail d'historien) ce qui s'est réellement passé et, ensuite, comprendre pourquoi ça s'est passé comme ça pour pouvoir en tirer les enseignements et, au final, progresser... ".

Cette démarche de détection et de correction des erreurs suppose le partage d'un certain nombre de prérequis de la part des acteurs. En particulier, l'observation des erreurs des autres permet de se corriger soi-même et la présence des collègues lors du débriefing est non seulement la garantie de repérer l'ensemble des erreurs commises sans risque de " passer à côté ", mais elle permet aussi d'envisager un éventail complet de solutions/corrections. Le partage de ces prérequis favorise la mise en place d'un processus constructif permettant d'améliorer ses propres pratiques à partir des critiques et des commentaires formulés par les autres. Ce processus d'apprentissage par l'erreur se fonde sur l'expérimentation et la pratique au quotidien. Comme le précise un pilote de l'une des équipes de présentation :

« Nous sommes dans le pilotage pur, où les sensations sont essentielles. Vous ne trouvez pas la réponse à vos problèmes dans une base de données! La connaissance théorique, c'est bien... Mais elle n'a pas de valeur, tant qu'elle n'est pas mise en pratique!"

La démarche de correction des erreurs en vol ou au sol (l'aspect mécanique) se caractérise par la nature expérientielle du savoir qu'elle permet de transmettre. Les escadrons de présentation poussent cette logique d'expérimentation à l'extrême en s'impliquant corporellement dans l'apprentissage. Lors des briefings, leurs pilotes répètent leur vol avant de prendre les commandes de leur avion. Les yeux fermés, ils réalisent, avec leurs mains, leurs doigts, leur tête et tout leur corps (pour les pilotes de l'équipe de voltige) l'ensemble des figures et des acrobaties qu'ils présenteront lors de l'exhibition. Cette « musique » un peu particulière traduit chacun des gestes techniques appris, critiqués et réappris lors des séances de débriefing. Elle représente également un moment d'intense concentration permettant aux pilotes de canaliser leurs émotions (telles que le stress) et de se préparer corporellement et sensoriellement à leur vol.

Le second objectif du briefing-débriefing cité par les interviewés est relatif à la progression du collectif. En effet, les pilotes et les navigateurs insistent sur le fait que la détection/confrontation des erreurs et la capacité d'en tirer des leçons au niveau individuel rejaillissent sur le groupe. Que les membres soient navigants ou non (les mécaniciens, par exemple), l'essentiel est qu'ils participent à la préparation et à l'exécution de la mission. Un pilote de transport nous explique :
« Le but ultime, c'est de progresser, de s'améliorer. Et quand je dis : "de s'améliorer », c'est volontairement impersonnel, parce que c'est pour tout le monde, pour tous les acteurs qui ont été présents : les contrôleurs, les pilotes, les navigateurs, les équipages, les mécaniciens... Pour tous les acteurs de la mission, le but est de progresser. Le but est que le débriefing conduise à des enseignements individuels ou de groupe, pour que, la prochaine fois, la machine soit encore plus performante... la machine au sens large : l'appareil complexe [constitué] de tous les individus ".

Chaque mission est considérée comme une opportunité d'apprentissage et d'expérimentation. En acceptant de travailler sur le mode de la critique constructive, chaque personnel aborde le débriefing comme un processus d'apprentissage multiniveaux qui lui permet de progresser non seulement en tant qu'individu, mais aussi en tant que membre d'une équipe. Ainsi, un pilote de chasse insiste sur le fait que :

«Le débriefing nous permet de nous re-préparer à refaire comme il faut, la prochaine fois. II faut vraiment voir ça comme un ensemble. Donc, le débriefing, à lui tout seul, ... il ne faut pas le sortir de son contexte. C'est dans cette logique de performance que nous travaillons, pour tirer les enseignements qui nous permettront, [nous] le groupe et l'individu, d'être performants. Les enseignements du débriefing vont permettre d'alimenter la connaissance globale et la connaissance individuelle. Ce n'est pas un exercice de style, c'est pour apprendre à être là au bon moment, le jour où il [le] faudra... ».

Selon les personnels interviewés, cette vision partagée de l'erreur comme opportunité d'apprentissage individuel et collectif repose sur un principe institutionnalisé au sein de l'Armée de l'air, la dépénalisation de l'erreur. Dès 2006, le chef d'état-major de l'Armée de l'air l'instaure officiellement :

« Nous devons accepter de rendre systématiquement compte de nos erreurs, de commenter et d'informer nos pairs de tous ces incidents évités au sol ou en vol, de ces événements où " c'est passé près ". Nous devons faire profiter, dans un but didactique, l'ensemble de notre communauté de toutes les expériences, aussi désagréables soient-elles " (source : ministère de la Défense).

Dans ce cadre, les pilotes sont engagés dans un processus d'apprentissage institutionnalisé gouverné par les règles de la transparence et de la reconnaissance des erreurs.

Les artefacts technologiques : se confronter à ses erreurs et interagir avec les autres

L'analyse des données met en lumière le rôle joué par les technologies. Ainsi, par exemple, l'usage des systèmes de reconstitution des vols exploités par les escadrons de chasse confronte systématiquement et objectivement les pilotes et les navigateurs à la réalité : le visionnage des enregistrements leur permet de se représenter avec précision le déroulement de la mission et ses éventuelles défaillances. De leur côté, 
les escadrons de présentation bénéficient d'un cameraman qui filme chacune de leurs sorties en vol et qui remet ensuite la vidéo aux pilotes :

« Le film de l'intégralité du vol est visionné par le pilote, une fois [celui-ci] descendu de l'avion. Pendant le vol, je [dit un autre pilote de l'équipe] suis à côté du cameraman et je fais des commentaires et des critiques, qui sont tous enregistrés. Ces commentaires sont écoutés par le pilote, une fois son vol terminé, assis devant son film. II scrute sa prestation, observe les erreurs qu'il a commises et [il] doit également se rendre compte de ce qu'il fait bien. Pourquoi a-t-il réussi cette figure? Ou pourquoi a-t-il "échoué "? Des questions auxquelles il doit obtenir des réponses. II doit connaître son état d'esprit durant ces moments..." .

Les faits sont montrés, la transparence est la règle et chaque participant doit être prêt à accepter d'assumer la pleine responsabilité de ses erreurs. En cela, les technologies ont quelque peu révolutionné les procédures de briefing-débriefing : avant leur introduction, certains pilotes expérimentés pouvaient avoir tendance à sous-estimer la gravité de leurs erreurs. Comme un navigateur d'un escadron de transport nous l'indique :

«Avant, il était très facile, pour un ancien [pilote], de dire qu'il avait fait ci, fait ça, que c'était lui qui était en meilleure position, etc. Et, finalement, le jeune en instruction, même s'il pouvait avoir des doutes, hé bien, il se référait à la parole du plus ancien, donc forcément du plus fort, ou du moins de celui qui parlait le plus fort. Or, le système de restitution a permis, justement, à ces gens-là de se remettre davantage en question. Ça, c'est une bonne chose... "

En permettant aux pilotes et aux navigateurs d'examiner les données objectives du vol, les systèmes de reconstitution et les films des vols favorisent le contrôle et la confrontation à la réalité. Comme nous l'explique un pilote de chasse, chacun, confronté à son vol, doit être capable de décrire avec précision les problèmes auxquels il a été confronté, d'expliciter les raisons qui l'ont conduit à faire une erreur et de tirer les leçons de cet incident pour ses prochains vols. Ce pilote précise :

"Nous savons tous - même les plus expérimentés d'entre nous - que les erreurs ne sont rien d'autre qu'une opportunité d'apprendre. Moi, ce qui m'a le plus marqué, dans ma vie professionnelle, c'est des phases où j'ai fait des erreurs qui m'ont été reprochées sèchement au cours du débriefing - preuves vidéo à l'appui ! Depuis, je ne les ai plus refaites, et j'y pense encore tellement... : ça m'a marqué... »

Une des équipes de présentation de l'Armée de l'air a mis en place en 2012 un dispositif de caméras installé dans le cockpit de ses avions. La plupart du temps, ces caméras sont dirigées vers les pieds et/ou les mains du pilote. L'objectif est d'accéder aux « façons de faire », en situation. Lors du visionnage des enregistrements vidéo, l'ensemble des pilotes est présent afin d'observer à la fois les erreurs techniques, les sources de problèmes (voire d'incident ou d'accident) en vol et les bonnes pratiques gestuelles et visuelles sources de réussite. II s'agit là d'un débriefing de groupe sur la prestation d'un seul. Comme l'explique un des pilotes :

" À partir de ces films intérieurs, nous constatons la façon dont [le collègue] pilote. Par exemple, le circuit visuel est très important en voltige et, souvent, les pilotes ne savent pas où regarder. Ils ont du retard dans le circuit visuel, ce qui leur fait mal déclencher leurs mouvements, de façon précipitée. II suffirait qu'ils aient une ou deux secondes d'avance sur leur circuit visuel pour qu'ils voient l'objectif arriver et déclenchent avec plus de vivacité : avec la caméra intérieure, on peut comprendre pourquoi l'anticipation est mal opérée..." ".

Le débriefing à partir des vidéos extérieures est intentionnellement dirigé vers la progression de l'équipe non seulement sur le plan technique, mais également collectif. II s'agit de construire une synergie dans le groupe, de favoriser l'apprentissage mutuel par les pratiques des autres. À ce sujet, un des pilotes interviewés insiste dans ces termes:

«Durant le débriefing de la vidéo intérieure, parfois, les critiques sont lourdes... Mais on doit surtout savoir s'encourager : tout doit être fait dans un esprit constructif. Sinon, ce n'est même pas la peine : le collectif sera nul, et les résultats également. »

II s'agit de partager ses savoir-faire en ayant pour volonté de faire progresser l'équipe dans son ensemble. Ce pilote poursuit :

"Le collectif se construit autour d'un équilibre un peu particulier, en fait : il y a l'esprit de compétition, très individualiste, qui nous conduit à vouloir être le meilleur pilote. Puis il y a l'esprit collectif, [qui est] essentiel pour la survie de l'escadron, de l'équipe. C'est ici que la vidéo [intérieure] joue son rôle : avec elle, on montre son savoir-faire et on communique ses " trucs" pour continuer à progresser en tant que collectif, en diffusant les pratiques, la façon dont on réussit telle ou telle figure, les erreurs à ne pas commettre... : le débriefing vidéo nous permet de doser tout ça... »

\section{Comportements et valeurs sociales : réflexivité et socialisation}

La grande majorité des interviewés considère que les séances de briefing-débriefing à la fois reposent et développent des qualités et des attitudes personnelles. Les plus fréquemment citées sont l'humilité et la réflexivité. Les pilotes et les navigateurs définissent l'humilité comme la capacité de reconnaître ses erreurs et d'en accepter l'entière responsabilité :

« Etre seul dans son avion, c'est une chose... Débriefer devant les autres pilotes, ses pairs - voire devant un escadron, lorsqu'il y a eu un souci au niveau de la sécurité des vols, c'est aussi prendre ses responsabilités devant tout le monde : il faut assumer... C'est nécessaire de débriefer, pour pouvoir progresser. Et c'est aussi un moyen d'être humble dans son travail... ».

Comme le précise un pilote d'une des équipes de présentation, I'humilité est certes une disposition 
personnelle, mais elle s'acquiert également au fil des débriefings :

«Souvent, on dit que la première année, c'est la plus dure, parce qu'effectivement, on se fait " briser " : on est très critiqué... Mais c'est constructif, car le but, c'est de nous permettre de reconnaître nos erreurs, pour [pouvoir] progresser. Ça y va un peu fort, parfois, parce que, bien sûr, tous nos vols ne sont pas parfaits..., loin de là !... ».

L'humilité est étroitement liée à la réflexivité, qui évoque cette capacité des participants au débriefing, à prendre du recul par rapport à leurs actions et à faire évoluer leurs pratiques en fonction de ce qu'ils ont appris. C'est ce qu'explique un ancien leader de la Patrouille de France :

" Le débriefing est directement associé à l'autoanalyse. Ce qui est essentiel, en tant que leader, c'est de savoir reconnaitre ses erreurs et [de] se questionner. Moi, je sais que, lorsque je constatais que durant trois vols d'affilée mon intérieur droit [pilote effectuant sa première année au sein de la Patrouille et évoluant à la droite du leader] n'arrivait pas à faire telle ou telle figure, je me disais que cela venait aussi de moi, tout simplement parce que c'est sur moi qu'il prenait ses repères! Qu'est-ce que cela implique ? Que je devais me remettre en question, en permanence. Ce n'est pas parce que l'on est leader que l'on sait tout faire. Au contraire, on découvre pas mal de choses. Même si l'on a un certain background, une vraie expérience, il faut aussi arriver à s'auto-analyser et à se remettre en question ».

Les séances de briefing-débriefing impliquent de la part des personnels navigants de savoir reconnaître leurs erreurs et d'en assumer la responsabilité pour éviter de les reproduire lors d'un prochain vol. Le débriefing est souvent décrit par les plus anciens comme une opportunité de socialisation, et par les plus jeunes comme un moyen d'intégration dans la communauté. Les personnels que nous avons interviewés ont souligné le rôle joué par le bar d'escadron (ou la salle de détente) dans la procédure de briefing-débriefing. Au sein d'un environnement convivial, les pilotes parlent librement de leur journée, de la pression qu'ils ont ressentie, des situations qu'ils ont vécues et des solutions qu'ils ont trouvées pour régler (ou pour contourner) des problèmes. En d'autres termes, ils débriefent à nouveau, mais d'une façon très informelle, sans le poids de la hiérarchie et sans la tension liée à l'évaluation. Comme nous le rapporte un mécanicien de l'une des équipes de présentation :

« II faut trouver des moments pour débriefer de façon plus détendue, moins formelle. Le bar d'escadron, par exemple, est essentiel en tant qu'espace convivial de discussion et de débriefing. On parle toujours de la mission, mais différemment, en partageant ses expériences, en prenant du recul sur ce qui vient de se passer. Ça permet aussi de connaître l'autre, au-delà de ses capacités techniques et de ses expertises, de mieux comprendre sa personnalité, ses attitudes. C'est aussi par ce biais-là que l'on crée un esprit d'équipe... ».
Ces discussions conduisent à la construction progressive d'un stock de connaissances communes qui permet aux personnels navigants d'acquérir une compréhension partagée des fondements et des pratiques du métier. Un pilote de transport nous explique ainsi :

« Je pense que le bar d'escadron participe à l'acquisition des connaissances, les vieilles histoires, les commentaires... II y a une manière de faire. En aéronautique, même militaire, il y a une manière globale. Et ça, c'est tellement complexe, qu'au début, on n'[en] a vraiment qu'une vague idée. Donc, il vaut mieux être au bar, à écouter ce qui s'est passé. Les gens qui ont été là-bas, qui se sont posés sur tel ou tel terrain... On va en retenir une manière de faire..., un sens du break pour se poser. II n'y a pas de barrières ". Ce faisant, le briefing-débriefing formel et les discussions informelles au bar d'escadron constituent un système social structuré et démocratique à partir duquel les participants partagent et diffusent les valeurs et la culture du débriefing. D'abord acquises en formation initiale avant d'être appropriées en escadron, ces valeurs deviennent une seconde nature pour les personnels navigants. En ce sens, la procédure de briefing-débriefing encourage une forme de standardisation culturelle des comportements et des attitudes. En particulier, il produit des dispositifs collectifs " facilitateurs ", tels que le contrôle social, la confiance mutuelle et la cohésion. Un pilote de chasse indique :

«Le bar d'escadron, ça permet aussi de gagner en capital confiance. Quelqu'un qui est un très bon professionnel, mais qui a un comportement déplorable au sol, qui ne sait pas se tenir - alors qu'en vol, il est à sa place..., il n'a pas les qualités requises pour être un bon pilote. C'est le rôle de l'éducation, de l'acculturation : c'est un tout. Dans son domaine de responsabilité, le débriefing, c'est faire le café le matin, par exemple. C'est savoir être à l'écoute, etc. C'est une progression, professionnelle mais également extra-professionnelle....

Les valeurs et les normes culturelles sont également véhiculées par les traditions au sein des escadrons. Ces dernières concrétisent l'appartenance à un escadron tout en participant à la construction d'une équipe unie et avançant dans la même direction. Un pilote de l'une des deux équipes de présentation de l'Armée de l'air nous explique ainsi que :

"La cohésion, pour moi, c'est une clé de voûte de la synergie collective. Et ça passe par les traditions. Le but, c'est de réunir les gens... Qu'ils se retrouvent dans des valeurs et des principes communs. Pour ça, on a des traditions qui nous ont été léguées par nos anciens. Les traditions, c'est ce qui pimente l'esprit d'équipe. Donc, forcément, ça renforce la synergie. II y a des petites traditions, tout au long de l'année : par exemple, la boucle à huit [lorsque les huit pilotes de la Patrouille de France réalisent une boucle de façon synchronisée] : après la première boucle à huit réussie, c'est à ce moment-là que les trois nouveaux pilotes ayant intégré la Patrouille de France vont recevoir leur insigne. En général, ils se voient remettre, à cette occasion, leur casque nominatif aux couleurs de l'équipe... ». 


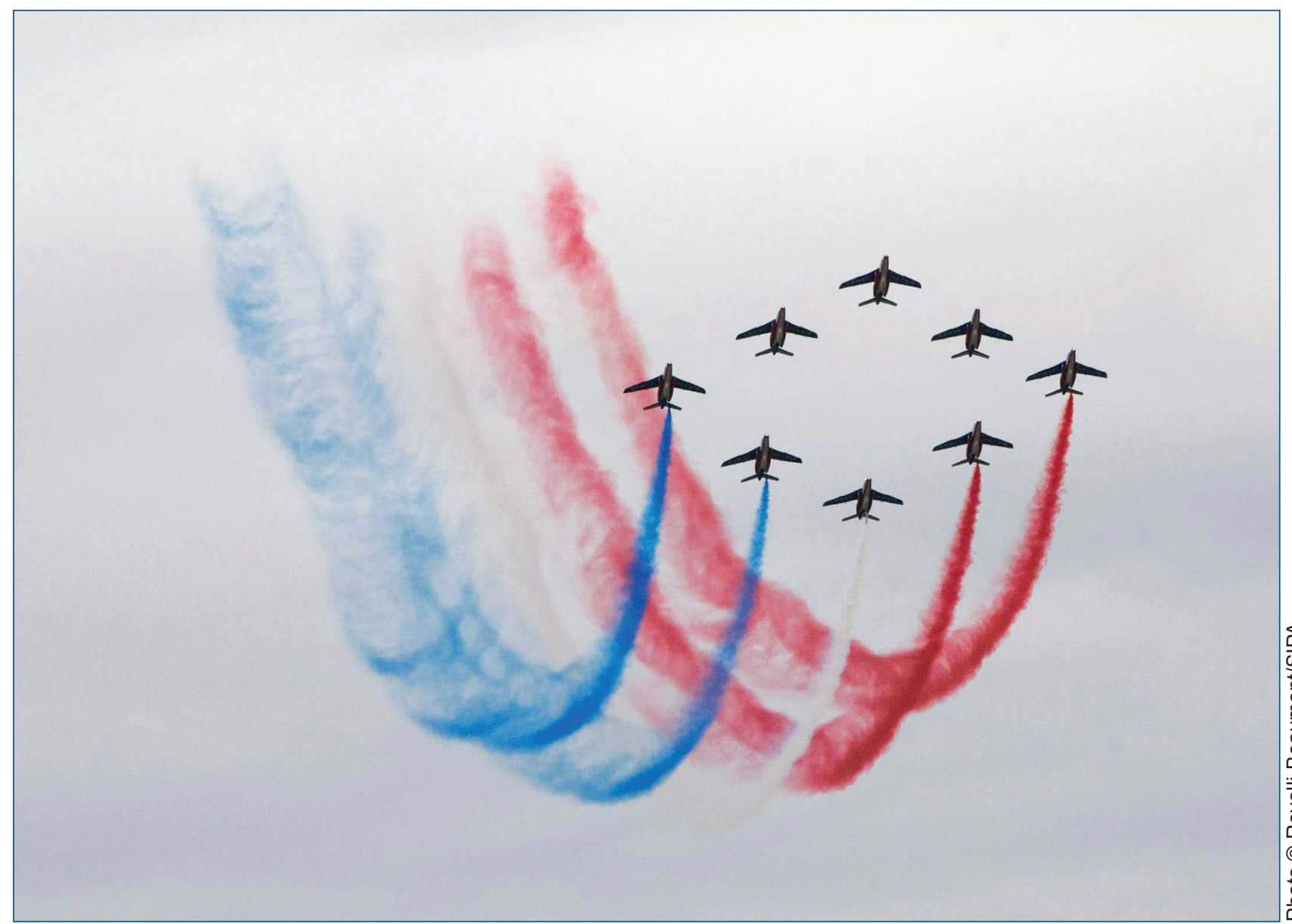

La Patrouille de France au Salon du Bourget, juin 2008.

« II y a des petites traditions, tout au long de l'année : par exemple, la boucle à huit [lorsque les huit pilotes de la Patrouille de France réalisent une boucle de façon synchronisée] : après la première boucle à huit réussie, c'est à ce moment-là que les trois nouveaux pilotes ayant intégré la Patrouille de France vont recevoir leur insigne. »

\section{Implications et conclusion}

En étudiant le quotidien des équipages navigants de l'Armée de l'air française, cet article confirme que les participants aux séances de briefing-débriefing développent leurs capacités d'apprentissage à partir de l'analyse réflexive et de la discussion critique des actions réalisées au cours de la mission. Conformément aux principes de l'approche pragmatiste, les acteurs apprennent d'autant mieux qu'ils réfléchissent aux actions entreprises individuellement et collectivement et qu'ils analysent les causes et les conséquences de leurs actions et de leurs erreurs, pour eux-mêmes et pour leur équipe.

L'analyse de cas permet également de comprendre les conditions selon lesquelles l'apprentissage dans et par l'action se construit, démontrant qu'il repose sur une architecture composée de trois catégories de ressources : des mécanismes d'apprentissage et des systèmes technologiques de communication et de contrôle social, qui s'articulent eux-mêmes autour d'un ensemble de valeurs culturelles et d'attitudes compor- tementales. L'articulation des trois catégories de ressources encourage l'enquête réflexive au sein des équipes. En ce sens, cette contribution s'inscrit dans la continuité des recherches antérieures effectuées sur les procédures pragmatistes d'apprentissage, tout en proposant une grille d'analyse opératoire des modes de gestion des barrières techniques, socioculturelles et psychologiques pesant sur le processus de briefingdébriefing (voir le Tableau 3 de la page suivante).

On observe, de plus, que les dispositifs d'identification et d'analyse des erreurs (technologies et films vidéo), qui pourraient être considérés comme particulièrement intrusifs par nombre d'individus, sont utilisés par des équipes partageant les valeurs culturelles du dialogue et de la remise en question de collectifs qui, par ailleurs, s'expriment au sein d'espaces d'interaction favorisant le « parler vrai » et des débats ouverts (le bar d'escadron, dans notre cas). Ainsi, ces dispositifs sont enactés dans un environnement favorable à l'apprentissage réflexif. Dans cette optique, nous comprenons que les barrières techniques, socioculturelles et psychologiques ne 


\begin{tabular}{|c|c|c|}
\hline $\begin{array}{c}\text { Facteurs } \\
\text { défavorables }\end{array}$ & Illustrations & Solutions apportées par le briefing-débriefing \\
\hline Techniques & $\begin{array}{l}\text { Complexité } \\
\text { des systèmes, } \\
\text { compétences } \\
\text { absentes ou } \\
\text { insuffisantes }\end{array}$ & $\begin{array}{l}\text { Formation (initiale et continue) et acculturation des acteurs aux } \\
\text { outils, principes et valeurs de l'apprentissage par et dans l'action. } \\
\text { Standardisation des pratiques du briefing-débriefing. } \\
\text { Intégration des phases de préparation, d'action et d'analyse } \\
\text { après action (favorable à l'expérimentation et à la réduction de la } \\
\text { complexité des tâches). } \\
\text { Systèmes techniques et technologiques favorisant la détection et } \\
\text { l'analyse des erreurs. }\end{array}$ \\
\hline Socioculturels & $\begin{array}{l}\text { Pénalisation de } \\
\text { l'erreur, absence de } \\
\text { culture du dialogue, } \\
\text { peu de réflexivité, } \\
\text { dévalorisation de } \\
\text { l'enquête }\end{array}$ & $\begin{array}{l}\text { Consonance entre les valeurs individuelles et les normes sociales } \\
\text { de l'organisation. } \\
\text { Rôle des cadres intermédiaires qui incarnent le rapport positif de } \\
\text { l'organisation face à l'erreur (dépénalisation). } \\
\text { Espaces de socialisation encourageant le dialogue et la réflexivité. } \\
\text { Compréhension et respect des traditions, des rituels, des } \\
\text { références historiques de l'organisation. }\end{array}$ \\
\hline $\begin{array}{l}\text { Psychologiques et } \\
\text { émotionnels }\end{array}$ & $\begin{array}{l}\text { Stress, peur, } \\
\text { anxiété, culpabilité, } \\
\text { insécurité, mauvaise } \\
\text { estime de soi }\end{array}$ & $\begin{array}{l}\text { Systèmes techniques et technologiques favorisant la transparence } \\
\text { et le sentiment de sécurité des acteurs. } \\
\text { Attribution des responsabilités et valorisation des individus } \\
\text { (estime de soi, minimisation du sentiment de culpabilité). } \\
\text { Modes de gestion sensoriels des émotions individuelles. }\end{array}$ \\
\hline
\end{tabular}

Tableau 3 : Les apports du briefing-débriefing pour lever les barrières à l'apprentissage organisationnel.

peuvent être levées par l'unique fait d'une décision managériale « extérieure » au contexte d'apprentissage. Le rôle de l'organisation est de venir en soutien des collectifs dans leur démarche d'apprentissage. Par exemple, elle peut introduire des systèmes de gestion des données qui facilitent la détection des anomalies et garantissent la disponibilité d'une expertise en matière d'analyse (CANNON et EDMONDSON, 2005). II peut également s'agir, pour les managers, de renforcer le sentiment de sécurité à travers la mise en place de systèmes valorisant le reportage des erreurs (par exemple, l'institutionnalisation de la dépénalisation de l'erreur, dans le cas qui nous occupe). Enfin, une attention particulière doit être apportée à la formation et à la communication interne visant à présenter les erreurs comme des opportunités d'apprentissage.

Si nos résultats contribuent à affiner notre compréhension des conditions selon lesquelles l'apprentissage dans et par l'action se construit, ils ne peuvent être généralisés. Une telle généralisation nécessiterait la collecte de données empiriques supplémentaires (études de cas multiples) et/ou l'adoption d'une approche quantitative. Or, nous avons choisi d'explorer un cas unique. Les implications théoriques et managériales restent donc fortement liées au terrain. Pour autant, ce travail ouvre des pistes de réflexion pour de futures recherches. En particulier, nous envisageons d'étudier comment les organisations appréhendent les facteurs défavorables à l'apprentissage organisa- tionnel en explorant d'autres procédures pragmatistes (apprentissage-action en environnement virtuel). Cette démarche permettrait d'adopter une posture critique (réflexive !) en vue de discuter la validité et la pertinence de nos résultats.

\section{Bibliographie}

ARGYRIS (C.) \& SCHÖN (D.), Organizational Learning: A Theory of Action Perspective, Reading MA: AddisonWesley, 1978.

BES (M.P.), « La capitalisation active des connaissances, principes, contextes et obstacles ॥, Gérer et Comprendre, $\mathrm{n}^{\circ} 54$, décembre 1998, pp. 38-51. http://annales.com/gc/1998/gc12-98/38-51.pdf

CANNON (M.) \& EDMONDSON (A.), "Failing to learn and learning to fail (intelligently)", Long Range Planning, vol. 38, 2005, pp. 299-319.

CARMELI (A.), "Social capital, psychological safety and learning behaviours from failures in organizations", Long Range Planning, vol. 40, 2007, pp. 30-44.

CATINO (M.) \& PATRIOTTA (G.), "Learning from errors: Cognition, emotions, and safety culture in the Italian Air Force", Organization Studies, vol. 34, n4, 2013, pp. 437-467.

DEWEY (J.), Logique : la théorie de l'enquête, New York, Henry Holt \& Company, 1938. 
EDMONDSON (A.), "The local and variegated nature of learning in organizations", Organization Science, vol. 13, n², 2002, pp. 128-146.

EISENHARDT (K.), "Building theories from case study research", Academy of Management Review, vol. 14, $\mathrm{n}^{\circ} 4,1989$, pp. 532-550.

FROMIGUÉ (A.), GODÉ (C.) \& PICQ (T.), « Et si vous aussi, vous passiez en mode projet ? ", Harvard Business Review, 2015. Disponible en ligne: http://www.hbrfrance.fr/chroniques-experts/2015/07/ 7478-et-si-vous-aussi-vous-passiez-en-mode-projet/

GODÉ (C.), « Développer les compétences collectives à partir des pratiques de retour d'expérience " à chaud »: le cas de l'équipe de voltige de l'Armée de l'air ", Revue française de gestion, vol. 38, n²23, 2012, pp. 167-180.

GODÉ (C.) \& BARBAROUX (P.), "Towards an architecture of organizational learning: Insights from French military aircrews", VINE, The Journal of Information and Knowledge Management, vol. 42, n³/4, 2012, pp. 321-334.

GOODMAN (P.S.), RAMANUJAM (R.), CARROLL (J.S.), EDMONDSON (A.C.), HOFMANN (D.A.) \& SUTCLIFFE (K.M.), "Organizational errors: Directions and future research", Research in Organizational Behavior, vol. 31, 2011, pp. 151-176.

JAMES (W.), Essays in Radical Empiricism, New York: Longmans, Green, 1912.

KOLB (D.), Experiential Learning: Experience as a source of learning and development, Upper Saddle River, NJ: Prentice Hall, 1984.

LAROCHE (H.), « L'ingénieur, le manager et l'astronaute ", Gérer et Comprendre, n53, septembre 1998, pp. 69-77.

http://www.annales.org/gc/1998/gc09-98/69-77.pdf
LIPSHITZ (R.), POPPER (M.) \& FRIEDMAN (V.), "A Multifacet Model of Organizational Learning", Journal of Applied Behavioral Science, vol. 38, $\mathrm{n}^{\circ} 1,2002$, pp. 78-98.

ORMEROD (R.), "The history and ideas of pragmatism", The Journal of Operational Research Society, vol. 57, n8, 2006, pp. 892-909.

POPPER (M.) \& LIPSHITZ (R.), "Organizational learning: Mechanisms, culture and feasibility", Management Learning, vol. 31, n², 2000, pp. 181-196.

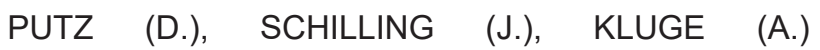
\& STANGENBERG (C.), "Measuring organizational learning from errors: Development and validation of an integrated model and questionnaire", Management Learning, vol. 45, n5, 2013, pp. 511-536.

REVANS (R.W.), The Origin and Growth of Action Learning, Brickley: Chartwell-Bratt, 1982.

RON (N.), LIPSHITZ (R.) \& POPPER (M.), "How organizations learn: Post-flight reviews in an F-16 fighter squadron”, Organization Studies, vol. 27, n8, 2006, pp. 1069-1089.

VAN DER VEGT (G.) \& BUNDERSON (J.), "Learning and performance in collective team identification", Academy of Management Journal, vol. 48, n³, 2005, pp. 532-547.

VAUGHAN (D.), The Challenger launch decision: Risky technology, culture, and deviance at NASA, The University of Chicago Press, Chicago, 1996.

YIN (R.), Case study research: Design and methods, Sage Publications, 2003.

ZHAO (B.), "Learning from errors: The role of context, emotion, and personality", Journal of Organizational Behavior, vol. 32, 2011, pp. 435-463. 